\title{
The circadian clock gene (Clock) regulates photoperiodic time measurement and its downstream process determining maternal induction of embryonic diapause in a cricket *
}

\author{
ShIN G. GOTO ID and Masatoshi NAGATA \\ Department of Biology and Geosciences, Graduate School of Science, Osaka City University, 3-3-138 Sugimoto, Sumiyoshi-ku, \\ Osaka 558-8585, Japan; e-mails: shingoto@osaka-cu.ac.jp, nagatamsts@gmail.com
}

Key words. Orthoptera, Trigonidiidae, Dianemobius nigrofasciatus, band-legged ground cricket, circadian clock gene, Clock, embryonic diapause, locomotor activity rhythm, photoperiodic time measurement, RNA interference (RNAi)

\begin{abstract}
In response to short days in autumn, most temperate multivoltine insects enter diapause, a state in which development or reproduction is suppressed or arrested, which serves to coordinate their development and physiology (or that of offspring) with annual changes in the environment (i.e. photoperiodism). This response is mediated by a measurement of time based on photoperiod (photoperiodic time measurement), which is thought to be regulated by a circadian clock. However, some studies also demonstrate the involvement of the circadian clock in an output process that generates phenotypes associated with diapause. To gain further insight into this, we silenced the Clock (Clk) gene, the main regulator of the circadian clock, in the band-legged ground cricket Dianemobius nigrofasciatus (Orthoptera: Trigonidiidae). Silencing the Clk gene using RNA interference (Clk RNAi) in female crickets resulted in abnormal circadian rhythms under constant darkness and light-dark conditions, thereby indicating the central role of this gene in the circadian clock mechanism. Clk RNAi females exhibited long-day oviposition behaviour, even when reared under short-day conditions, thereby indicating the involvement of $C l k$ in photoperiodic time measurement. In addition, Clk RNAi females immediately laid non-diapause-type eggs, which was not recorded in control females under either short-day or long-day conditions and cannot be explained in terms of dysfunction of photoperiodic time measurement. Accordingly, we speculate that Clk could also be involved in a downstream process that results in the laying of diapause-type eggs.
\end{abstract}

\section{INTRODUCTION}

Organisms have evolved a seasonal timing system that enables them to coordinate their development and physiology (or that of their offspring) with annual changes in the environment, using day length (photoperiod) as a cue (Tauber et al., 1986; Danks, 1987). This property is termed photoperiodism and an organism's response to photoperiod is referred to as a photoperiodic response. Organisms measure the length of night or day using a measurement of time based on photoperiod (photoperiodic time measurement). In this regard, the most well-established photoperiodic event in insects is diapause, where development or reproduction is suppressed or arrested in conjunction with an extensive shutdown of metabolic activity (Košt'ál, 2006). The stages at which diapause occurs are species-specific and diapause at embryonic, larval or nymphal, pupal and adult stages have all been described (Denlinger et al., 2012). The period sensitive to photoperiod, during which an insect receives photoperiodic cues that trigger the photoperiodic response, occurs in advance of diapause, the most extreme example of which is when this sensitive stage occurs in one generation and diapause in the next (i.e. a maternal effect) (Mousseau \& Dingle, 1991).

The circadian clock that regulates the rhythm of locomotor activity comprises at least a dozen circadian clock genes, including period (per), timeless (tim), mammaliantype cryptochrome (cry-m or cry2), cycle (cyc), Clock (Clk), Par domain protein 1 (Pdpl), vrille and clockwork orange (Tomioka, 2014; Tomioka \& Matsumoto, 2015; Patke et al., 2020). The principal mechanism associated with these genes is a transcription-translation feedback loop, which takes approximately $24 \mathrm{~h}$ to complete. Involvement of the circadian clock in photoperiodic time measurement was first proposed by Erwin Bünning in the 1930s (Bünning, 1936), and subsequently, further models were proposed, initially a modified version of Bünning's original model, referred to as the 'external coincidence model', and thereafter an alternative hypothesis, the 'internal coincidence

\footnotetext{
* This paper was contributed to a virtual special issue in memory of Ivo Hodek, a long-time editor of the European Journal of Entomology, who died on June 11, 2021, shortly after his ninetieth birthday.
} 
model'. Both these models assume the central role of the circadian clock in photoperiodic time measurement (Saunders, 2020). Molecular physiological studies have revealed the involvement of circadian clock genes in the photoperiodic response of a diverse range of insect species in the orders Orthoptera (Sakamoto et al., 2009), Heteroptera (Ikeno et al., 2010; Kotwica-Rolinska et al., 2017; Tamai et al., 2019), Coleoptera (Zhu et al., 2019), Hymenoptera (Mukai \& Goto, 2016), Lepidoptera (Mohamed et al., 2014; Iiams et al., 2019; Ikeda et al., 2021) and Diptera (Pegoraro et al., 2014; Meuti et al., 2015). For example, silencing the major clock genes per, cry-m, cyc and Clk results in the disruption of the circadian clock regulating the rhythm of cuticular deposition and photoperiodic regulation of reproductive diapause in the bean bug Riptortus pedestris (Ikeno et al., 2010, 2011a, b, 2013). Knock-down of per and tim in female cabbage beetles (Colaphellus bowringi) subjected to a diapause-inducing photoperiod resulted in a reduction in lipid content, a more typical non-diapause phenotype ( $\mathrm{Zhu}$ et al., 2019). Loss-of-function due to mutation of several circadian clock genes abolishes the photoperiodic response associated with reproductive output in the monarch butterfly Danaus plexippus (Iiams et al., 2019).

The findings of these studies thus provide compelling evidence that the circadian clock plays a pivotal role in controlling photoperiodic responses. Involvement of the circadian clock in photoperiodic time measurement is proposed on the basis of theoretical and empirical evidence (Saunders, 2020) and is well supported by the findings of genetic and molecular physiological studies (Pegoraro et al., 2014; Mukai \& Goto, 2016; Urbanová et al., 2016; Ikeda et al., 2021). Conversely, the circadian clock genes in question might regulate a downstream process in photoperiodic time measurement (Emerson et al., 2009; Bradshaw \& Holzapfel, 2010). Indeed, a noncircadian function downstream of photoperiodic time measurement is reported for the $C l k, c y c, c r y-m$ and $P d p 1$ genes in diapausespecific gene expression in the gut of Pyrrhocoris apterus (Bajgar et al., 2013a, b). A similar function of the circadian clock genes is also proposed in D. melanogaster and Drosophila triauraria (Saunders et al., 1989; Tauber et al., 2007; Yamada \& Yamamoto, 2011).

To gain further insight in this regard, we investigated the role of $C l k$, a main component of the circadian clock (Zhao et al., 2003; Kilman \& Allada, 2009), in photoperiod induced diapause in the band-legged ground cricket Dianemobius nigrofasciatus (Matsumura) (Orthoptera: Trigonidiidae). This species shows a clear maternal effect, in which females exposed to long-day photoperiods lay non-diapause-type eggs that develop into nymphs without interruption. In contrast, females maintained under a short-day photoperiod lay eggs that are destined to enter diapause, where development is arrested at the blastoderm stage (Goto et al., 2008; Tanigawa et al., 2009). When females are transferred from a short-day to long-day environment, they gradually reduce and increase the numbers of diapause- and non-diapause-type eggs laid, respectively, thereby resulting in a gradual reduction in the incidence of diapause (Kidokoro \& Masaki, 1978). Mother crickets monitor and assess photoperiod for several days and on the basis of this assessment determine the developmental trajectory of their offspring. Consequently, the incidence of diapause changes gradually. Thus, if the circadian clock controls photoperiodic time measurement, it is likely that dysfunction of the clock would be manifested by a gradual change in the incidence of diapause. In contrast, if the clock controls a downstream process in photoperiodic time measurement, it is assumed that the effects of dysfunction on the operation of the clock would be observed immediately.

In the present study, we initially cloned the $C l k$ gene from $D$. nigrofasciatus and recorded its expression. Thereafter, we investigated the role of $C l k$ in the rhythm of cricket locomotor activity, and subsequently examined its role in the photoperiodic induction of diapause. Our findings reveal that $C l k$ is involved not only in photoperiodic time measurement but also in a downstream process resulting in production of diapause-type eggs.

\section{MATERIALS AND METHODS}

\section{Insects}

From the end of June to the end of July in 2011 and 2012, adults and nymphs of $D$. nigrofasciatus were collected from a grassy field on the campus of Osaka City University $\left(34^{\circ} 59^{\prime} \mathrm{N}\right.$, $\left.135^{\circ} 50^{\prime} \mathrm{E}\right)$. Approximately $30-50$ insects were reared in a plastic container ( $80 \mathrm{~mm}$ diameter, $140 \mathrm{~cm}$ height) at $25.0 \pm 1.0^{\circ} \mathrm{C}$ under long-day conditions (16L : 8D), during which time, they were fed an artificial insect diet (Oriental Yeast, Tokyo, Japan) and pieces of fresh carrot under long-day conditions. Moist cotton was provided as a source of water and also served as a substrate for oviposition. The eggs were placed on a piece of moist cotton in plastic dishes $(50 \mathrm{~mm}$ diameter, $12 \mathrm{~mm}$ depth), which were thereafter maintained in an airtight container (150 mm diameter, $90 \mathrm{~mm}$ depth).

\section{Cloning of $\mathrm{Clk}$}

To determine the nucleotide sequence of $C l k$ in D. nigrofasciatus, RNA was extracted from the whole body of a field-collected male cricket, using Trizol Reagent (Thermo Fisher Scientific, Waltham, MA, USA). cDNA was synthesised using an oligo(dT) $)_{12-18}$ primer and SuperScript III Reverse Transcriptase (Thermo Fisher Scientific) and was used as a template for PCR using the primer pair dnclk-f1 and -r1 (shown in Table 1) and Platinum Taq DNA Polymerase (Thermo Fisher Scientific) according to the supplier's instructions. The PCR amplification programme was as follows: an initial denaturation at $94^{\circ} \mathrm{C}$ for 1 min, followed by 45 cycles of $94^{\circ} \mathrm{C}$ for $30 \mathrm{~s}, 52^{\circ} \mathrm{C}$ for $30 \mathrm{~s}$ and $72^{\circ} \mathrm{C}$ for $1.5 \mathrm{~min}$. The amplified fragment was purified using a Wizard SV Gel and PCR Clean-Up System (Promega, Madison, WI, USA) and subcloned into a plasmid using TArget Clone Plus (Toyobo, Osaka, Japan). Following transformation into Escherichia coli DH5 $\alpha$ competent cells (Competent Quick DH5 $\alpha$, Toyobo), plasmids were purified using the Wizard Plus SV Minipreps DNA Purification System (Promega). Purified plasmids were sequenced using an ABI PRISM 3010 Genetic Analyzer (Thermo Fisher Scientific), a BigDye Terminator v3.1 Cycle Sequence kit (Thermo Fisher Scientific) and M13 Primers M4 and RV (Takara Bio, Kusatsu, Japan). 
Table 1. The primers used in the present study.

\begin{tabular}{|c|c|c|}
\hline Gene & Primer name & Nucleotide sequence (from 5' to 3') \\
\hline \multirow{8}{*}{ Clock (Clk) } & dnclk-f1 & AGA AGA AGC GGC GGG AYC ART TYAA \\
\hline & dnclk-r1 & GGT CTG CAG CCA GAT CCA YTG YTG \\
\hline & qpcr-DnClk-f1 & GGG ACC AGT TCA ACA TGC TTA TT \\
\hline & qpcr-DnClk-r1 & CAG TAG ATT TAT CCA TTT TGC GAC TAT T \\
\hline & dndsclk-f1 & GCT CGT CCA GTT TGT TGG TT \\
\hline & dnT7clk-f1 & GGA TCC TAA TAC GAC TCA CTA TAG GGC TCG TCC AGT TTG TTG GTT \\
\hline & dndsclk-r1 & GGA GGA GCT CTG TGG TCAAG \\
\hline & dnT7clk-r1 & GGA TCC TAA TAC GAC TCA CTA TAG GGG AGG AGC TCT GTG GTC AAG \\
\hline \multirow[t]{4}{*}{$\beta$-lactamase (bla) } & pGBetalacm-F1 & TCG CCG CAT ACA CTA TTC TC \\
\hline & pGBetalacmT7-F1 & GGA TCC TAA TAC GAC TCA CTA TAG GTC GCC GCA TAC ACT ATT CTC \\
\hline & pGBetalacm-R1 & TAC GAT ACG GGA GGG CTT AC \\
\hline & pGBetalacmT7-R1 & GGA TCC TAA TAC GAC TCA CTA TAG GTA CGA TAC GGG AGG GCT TAC \\
\hline Elongation factor-1 $\alpha$ & qpcr-DnEF1-f1 & TCA CGC TCA GCC TTC AGC TT \\
\hline$(E F-1 \alpha)$ & qpcr-DnEF1-r1 & GAT GGG AAA GGG TTC ATT CAA G \\
\hline
\end{tabular}

dnclk-f1 and -r1 were designed by Kamae et al. (2010).

\section{Reverse transcription-quantitative polymerase chain reaction (RT-qPCR)}

To analyse the diel pattern of $C l k$ expression, female crickets were reared under short-day conditions (12L : 12D) from the $1 \mathrm{st}$ instar and their heads were collected every $4 \mathrm{~h}$ (Zeitgeber times [ZTs] 2, 6, 10,14, 18, and 22, where ZT0 is defined as the time of light-on) on days 10-15 (day 0 was defined as the day of adult emergence). To observe the effect of gene silencing, heads of females that were reared under short-day conditions were collected at ZT6 on day 15 .

RNA was extracted using Trizol Reagent followed by purification with a PureLink RNA Mini kit (Thermo Fisher Scientific). cDNAs were synthesised using a High-Capacity cDNA Archive kit (Thermo Fisher Scientific) and relative abundance of the target cDNA was measured using a 7500 Real-Time PCR system (Thermo Fisher Scientific) with Go Taq qPCR Master Mix (Promega) according to the supplier's instruction. The sequences of the primers used are shown in Table 1. Elongation factor 1 alpha $(E F-1 \alpha)$ was used as a reference gene to quantify the relative mRNA levels and a standard curve was used to estimate the levels of mRNA in samples. PCR efficiencies for $C l k$ and $E F-1 \alpha$ were 1.01 and 1.07, respectively. R2 values of $C l k$ and $E F-1 \alpha$ standard curves were 0.994 and 0.995 , respectively.

\section{RNA interference (RNAi)}

Clk double-stranded RNA (dsRNA) (hereafter abbreviated as ds $C l k$ ) was synthesised using the T7 RiboMAX Express RNAi System (Promega) in accordance with the supplier's instruction. A template DNA fragment of $C l k$ was amplified using Pwo SuperYield DNA Polymerase (Roche, Basel, Switzerland) with the primer pairs dndsclk-f1 and -r1 and dnT7clk-f1 and -r1 (shown in Table 1). As a control, we also synthesised dsRNA of the bacterial $\beta$-lactamase (bla) gene (dsbla), which confers ampicillin resistance. The concentration of dsRNA was adjusted to $1 \mu \mathrm{g} \cdot \mu \mathrm{L}^{-1}$ with nuclease-free water. One-day-old adult female crickets that were reared under short-day conditions (12L : 12D) were anaesthetised on ice for $5 \mathrm{~min}$, injected with $1 \mu \mathrm{L}$ of dsRNA solution into the abdomen and maintained under short-day or long-day conditions.

\section{Recording locomotor activity}

For the purposes of recording cricket activity, we used adult females that had been maintained under short-day conditions. These individuals were individually housed in plastic chambers (50 $\mathrm{mm}$ in diameter, $15 \mathrm{~mm}$ in depth) and provided with unlimited food and water. As a means of assessing activity, we directed an infrared beam (EE SPW-321, Omron) across the chambers, and the number of interceptions of the beam was recorded at 6-min intervals on a personal computer (Shiga et al., 1999). The recording chambers were placed in an incubator maintained at $25^{\circ} \mathrm{C}$ and equipped with a $15-\mathrm{W}$ fluorescent lamp (FL 15W; Panasonic, Kadoma, Japan) with an irradiance of approximately $1.0-3.5 \mathrm{~W} \cdot \mathrm{m}^{-2}$. Crickets were maintained in constant darkness for 20 days and subsequently placed under short-day conditions. Rhythmicity was determined by chi-square periodogram analysis (Sokolove \& Bushell, 1978) and activity characterised by a clear single peak above the 0.05 confidence level between 20 and $28 \mathrm{~h}$ in the periodogram was considered to be rhythmic.

Given that we detected differences in the locomotor activities of individuals, we calculated the average daily activities under light-dark conditions. For each cricket, the numbers of recorded movements per $30 \mathrm{~min}$ were summed and the summed activity was divided by the mean 30-min activity of the individual. We further calculated the slope of the regression line from ZT6 to ZT11.5 to estimate scotophase anticipation.

\section{Assessment of diapause status}

Individual adult females were paired with a single adult male in glass tubes (15 mm in diameter, $100 \mathrm{~mm}$ in length) and the eggs laid by each female were collected daily. Non-diapausing eggs absorb water $6-8$ days after oviposition at $25^{\circ} \mathrm{C}$ and thereby the lengths of the major and minor axes of the egg became 1.1-1.3 times longer, while the diapause-destined eggs enter diapause prior to the absorption of water (Masaki, 1960; Goto et al., 2008). In the present study, we maintained eggs under long-day conditions and assessed diapause status on day 13 under a stereomicroscope. Eggs that had absorbed water were considered to be non-diapause, whereas those that did not were assumed to have entered diapause (Shiga \& Numata, 1997; Goto et al., 2008).

To clarify temporal changes in the incidence of diapause, we calculated the 5-day moving average of diapause incidence by dividing the number of diapause eggs by the total number of eggs laid in the 5 days and categorised the temporal patterns into five types (Fig. 1). Type 1: crickets mainly deposited diapause eggs throughout the experimental period and the incidence of diapause was invariably higher than $50 \%$. Type 2 : crickets initially deposited non-diapause eggs, but subsequently changed the offspring's developmental trajectory from non-diapause to diapause. Conversely, at a later stage, the crickets reversed the trajectory from diapause to non-diapause. In this pattern, the incidence of diapause is lower than $50 \%$ during the initial phase of the experimental period, becoming higher than $50 \%$ during the middle period, but lower than $50 \%$ at the end of the period. Type 3: crickets initially deposited non-diapause eggs, but later they changed the offspring's developmental trajectory to diapause, a strategy that 
Type 1

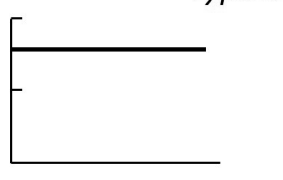

Type 2

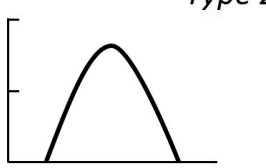

Type 3

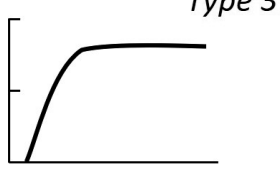

Type 4

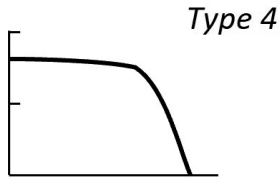$$
\begin{array}{ll}
\widehat{0} & 100 \\
0 & \\
\frac{0}{3} & 50 \\
\frac{0}{0} & \frac{\pi}{0} \\
\frac{0}{0} & 0
\end{array}
$$

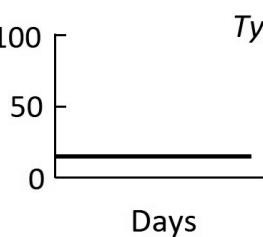

Fig. 1. Temporal profiles in the incidence of different types of diapause. Type 1: incidence is higher than $50 \%$ throughout the experimental period. Type 2: incidence is lower than $50 \%$ at the beginning of the experimental period, is higher than $50 \%$ during the middle period, and subsequently becomes lower than $50 \%$ at the end of the period. Type 3: incidence is lower than $50 \%$ at the beginning of the experimental period but becomes higher than $50 \%$ during the middle or end of the period. Type 4: initially incidence is higher than $50 \%$ but later becomes lower than $50 \%$. Type 5 : incidence is lower than $50 \%$ throughout the experimental period.

was maintained until the end of the experimental period. In this case, the incidence of diapause is lower than $50 \%$ at the beginning of the experimental period but becomes higher than $50 \%$ in the middle or final phase of the experimental period. Type 4: crickets initially deposited diapause eggs, but later they changed the offspring's developmental trajectory to non-diapause and maintained this strategy until the end of the experimental period. In this case, the incidence of diapause is initially higher than $50 \%$ but later becomes lower than 50\%. Type 5: crickets mainly deposited non-diapause eggs throughout the experimental period, and thus the incidence of diapause is consistently lower than $50 \%$.

\section{Statistical analyses}

A chi-square goodness-of-fit test was used to establish whether the data were drawn from a normal distribution. Bartlett's test was used to determine whether the samples had equal variance. Student's $t$-test and one-way ANOVA were used to analyse parametric data, and the Mann-Whitney $U$ and Steel-Dwass tests were used to analyse non-parametric data. (a)

(b)
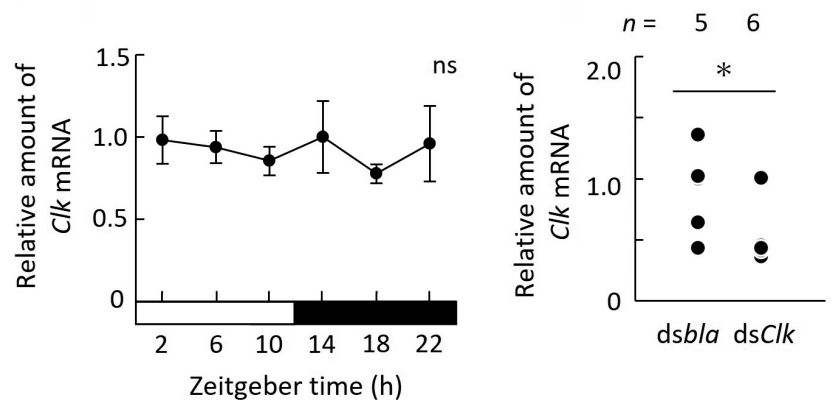

Fig. 2. Temporal profiles in the levels of $C l k$ mRNA and the effects of the injection of dsRNA on the levels of Clk mRNA in adult females of Dianemobius nigrofasciatus. (a) Diel patterns in the levels of $C / k$ mRNA (mean $\pm S D$ ) in the heads of females on days 10-15 under short-day conditions. The highest mean was set to 1.0. Horizontal open and closed bars indicate the photophase and scotophase, respectively. One-way ANOVA revealed no statistical significance (ns, $P \geq 0.05$ ). $n=4$ or 5 for each time point. (b) Effects of the injection of dsRNA on the levels of Clk mRNA in the heads of crickets at ZT 6 on day 15 under short-day conditions. The median value of the dsbla-injected crickets was set to 1.0. One-sided Mann-Whitney $U$-test detected a significant difference $\left({ }^{*} P<0.05\right)$. $n$, the number of samples. EF-1 $\alpha$ was used as a reference gene.

\section{RESULTS}

\section{Cloning}

cDNA of a partial $C l k$ sequence (877 bp) was obtained from D. nigrofasciatus (GenBank/EMBL/DDBJ accession number LC633975). A BLAST search of GenBank (Altschul et al., 1997) revealed that its putative amino acid sequence is very similar to that of CLK in other insects, particularly the CLK of the mangrove cricket (Apteronemobius asahinai) and two-spotted cricket (Gryllus bimaculatus) (data not shown). Analysis using the SMART programme (Letunic et al., 2021) revealed two PER-ARNT-SIM (PAS) domains in the sequence of the CLK of D. nigrofasciatus.

\section{Temporal pattern of $\mathrm{Cl} k$ expression and gene silencing}

Amounts of $C l k$ mRNA were normalised to those of $E F-1 \alpha$ mRNA, the amounts of which showed no statistical difference among samples (one-way ANOVA, $P>0.05$ ). We detected no significant variation in the diel amounts of Clk mRNA in the heads of crickets on days 10-15 (Fig. 2a; one-way ANOVA, $P>0.05$ ).

In female crickets maintained under short-day conditions, dsClk was injected on day 1 and $C l k$ mRNA levels in the head were quantified at ZT 6 on day 15 (Fig. 2b), which revealed that $C l k$ mRNA levels in the dsClk-injected females were lower than those in the control crickets (onesided Mann-Whitney $U$-test, $P<0.05$ ).

\section{Locomotor activity rhythms}

Locomotor activity was recorded under constant darkness (DD) for 20 days and subsequently under the lightdark (LD) cycles of a short-day photoperiod for 10 days (Fig. 3). Given that activity patterns of the crickets under DD gradually changed over time, we separately analysed activity during the initial (DD1) and subsequent (DD2) 10- 

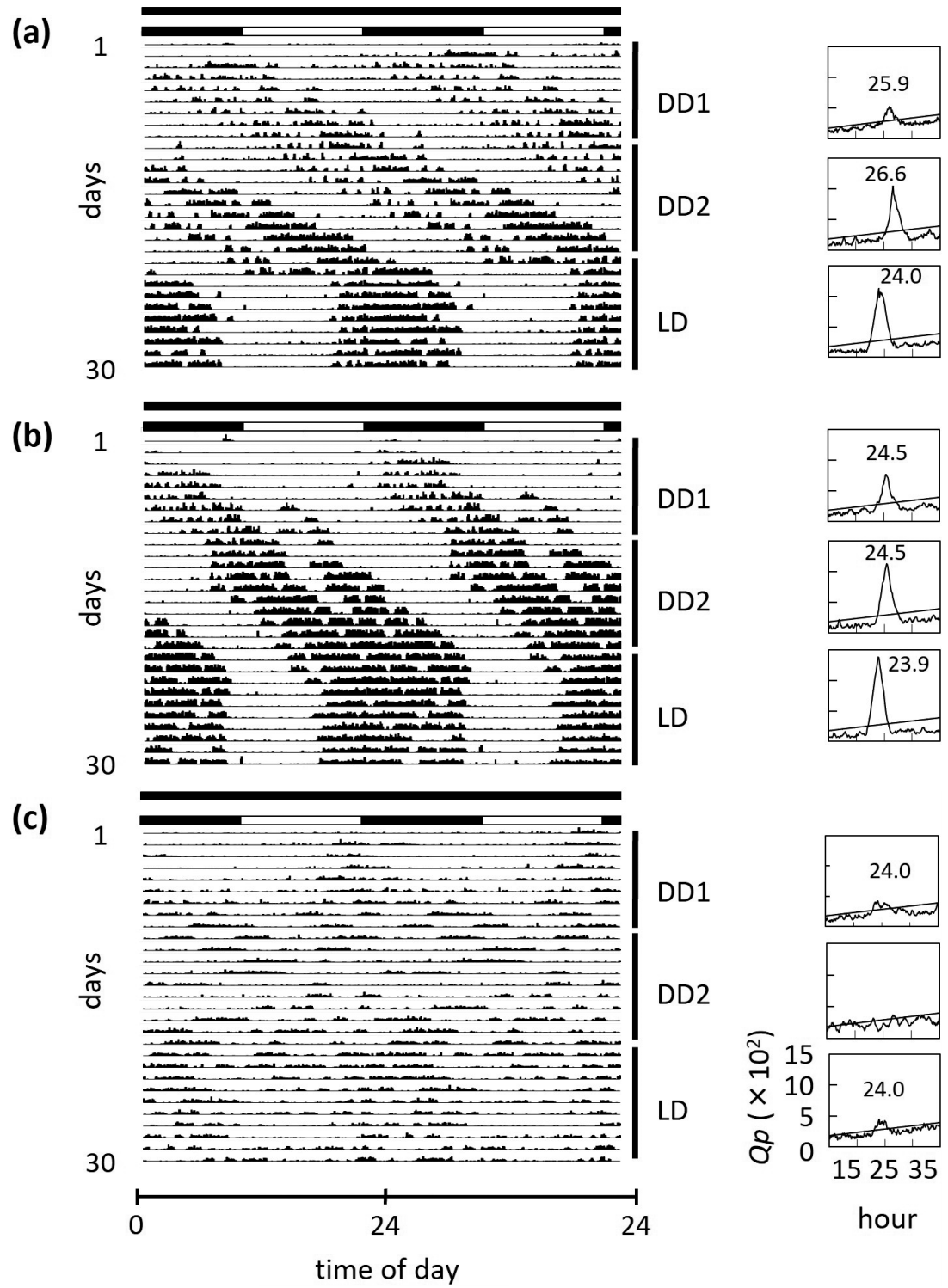

Fig. 3. Representative locomotor activity of intact (a), dsbla-injected (b) and dsClk-injected (c) females of Dianemobius nigrofasciatus. The activity was double plotted. Insects were maintained in constant darkness for 20 days (the initial 10-day period is termed DD1 and the following 10-day is termed DD2) and subsequently maintained under short-day light-dark conditions (LD) for 10 days. Open and closed bars on the graph indicate the photophase and scotophase, respectively. Chi-square periodograms are also shown.

day periods. All intact crickets (crickets that were not injected) $(n=10)$ showed clear rhythmic activity during both DD1 and DD2 (Fig. 3a and Table 2), with free-running periods of $25.1 \pm 1.0 \mathrm{~h}$ (mean $\pm \mathrm{SD}$ ) and $26.2 \pm 2.8 \mathrm{~h}$, respectively. One cricket died thereafter, whereas the remaining nine crickets showed clear rhythmic activity with a period of $24.0 \pm 0.0 \mathrm{~h}$ under LD. Similarly, all crickets injected with dsbla showed clear rhythmic activity under DD1 $(24.9 \pm 1.0 \mathrm{~h})$ and DD2 $(24.7 \pm 0.6 \mathrm{~h})(n=12$; Fig. $3 \mathrm{~b}$ and Table 2) and also showed clear rhythmicity under LD (24.0 $\pm 0.1 \mathrm{~h}$ ). However, whereas seven of 11 crickets injected with dsClk showed clear rhythmicity under DD1, five became arrhythmic under DD2 (Fig. 3c and Table 2). Only two of these 11 crickets showed clear rhythmicity under both DD1 and DD2, whereas four were arrhythmic under both DD1 and DD2 (Table 2). These patterns of locomotor activity were found to differ significantly between dsClk- injected and control crickets (Mann-Whitney $U$-test, $P<$ 0.05; Table 2) and tend to indicate that $C l k$ RNAi disrupts the function of the circadian clock under DD, although in some crickets, it may take more than 10 days before the effects of $C l k$ RNAi become apparent.

Three dsClk-injected crickets died during LD exposure so our analyses were based on the data obtained for the remaining eight crickets. In contrast to $\mathrm{DD}$, dsClk-injected crickets showed a clear rhythmic pattern under the subsequent LD, as observed in control crickets (Fig. 3). To examine these activity patterns in greater detail, we calculated the average daily activity profiles under LD (Fig. 4a), which revealed that most dsbla-injected crickets increased their activity during late photophase, whereas some of the dsClk-injected crickets failed to show such clear anticipation. To quantify their scotophase anticipation, we calculated the regression slopes of the activity increment from ZT6 
(a)

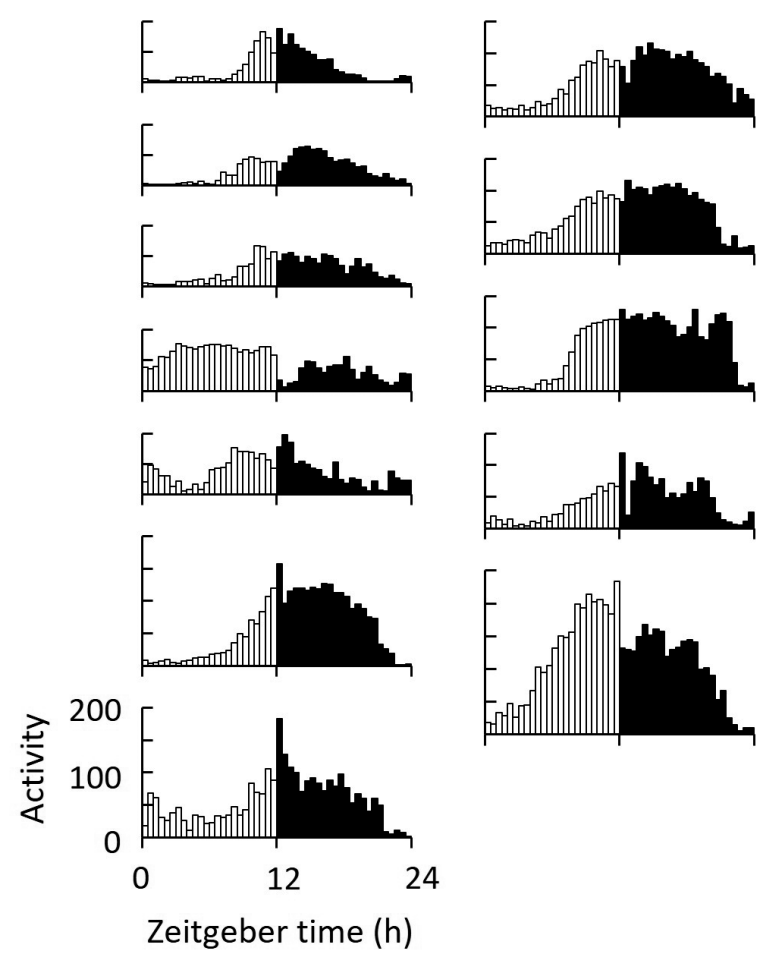

dsClk
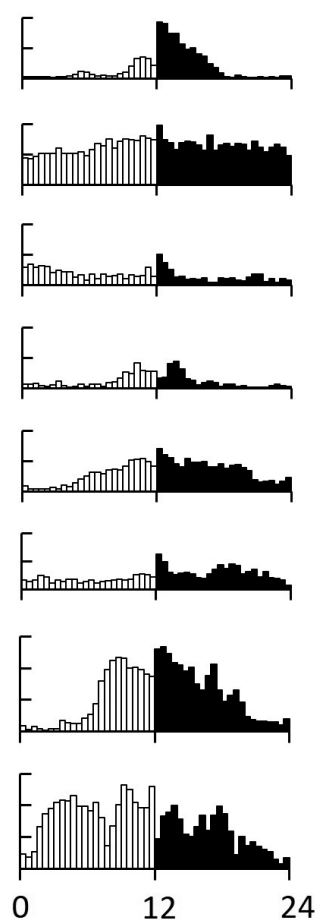

(b)

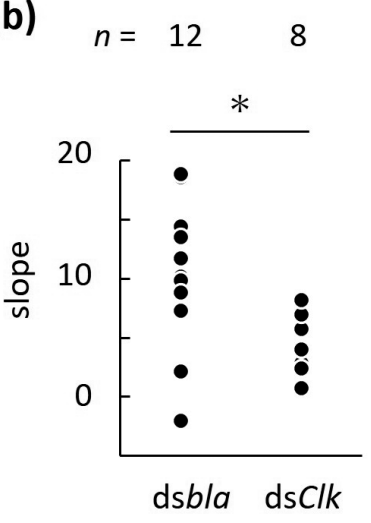

Fig. 4. Activity profiles (a) and scotophase anticipation (b) of dsbla-injected and dsClk-injected females of Dianemobius nigrofasciatus under light-dark conditions. (a) Average daily activity profiles of individual crickets. Open and closed columns indicate activity during the photophase and scotophase, respectively. (b) The slope of the regression line of the activity from ZT6 to 11.5 . Data were obtained from (a). Student's $t$-test detected a significant difference $\left({ }^{*} P<0.05\right) . n$ - number of samples.

(middle photophase) to ZT 11.5 (late photophase) (Fig. $4 \mathrm{~b})$, which revealed that the slopes obtained for dsClkinjected crickets were smaller than those of dsbla-injected crickets (Student's $t$-test, $P<0.05$ ). Thus, it is conceivable that the rhythm-like behaviour of Clk RNAi crickets under LD reflects a masking effect of light or, alternatively, that the runing of the circadian clock is perturbed under lightdark conditions.

\section{Photoperiodic regulation of diapause and the effects of Clk RNAi}

Photoperiodic regulation of the developmental trajectory of cricket offspring (diapause or non-diapause) is shown in Fig. 5. Intact females under short-day conditions deposited eggs destined to enter diapause throughout the experimental period, an oviposition pattern we categorised as Type 1 . Among crickets that were initially reared under short days

Table 2. The numbers of rhythmic and arrhythmic female crickets in the first (DD1) and the second (DD2) phases of constant darkness ${ }^{\S}$.

\begin{tabular}{|c|c|c|c|c|c|c|}
\hline Treatment & $n$ & $\frac{\mathrm{DD} 1}{\mathrm{DD} 2}$ & $\begin{array}{l}\text { Rhythmic } \\
\text { Rhythmic }\end{array}$ & $\begin{array}{l}\text { Rhythmic } \\
\text { Arrhythmic }\end{array}$ & $\frac{\text { Arrhythmic }}{\text { Arrhythmic }}$ & $\begin{array}{c}\text { Statistical } \\
\text { test }^{\#}\end{array}$ \\
\hline Intact & 10 & & 10 & 0 & 0 & $a$ \\
\hline dsbla & 12 & & 12 & 0 & 0 & a \\
\hline dsClk & 11 & & 2 & 5 & 4 & $b$ \\
\hline
\end{tabular}

\$Rhythmicity was determined using a chi-square periodogram in which activity characterised by a clear single peak above the 0.05 confidence level between 20 and $28 \mathrm{~h}$ in the periodogram was considered to be rhythmic. "Different letters indicate a significant difference (Mann-Whitney U-test, $\mathrm{P}<0.05$ ). and then transferred to long days on the day after adult emergence, four of seven deposited diapaus-type eggs at the beginning of the experimental period, although subsequently, they deposited non-diapause-type eggs (Type 4). The remaining three females laid non-diapause-type eggs from the beginning of the experimental period (Type 5). These results indicate that females deposit non-diapausetype eggs in response to long days, although the timing of the switch in oviposition strategy varied among individu-

Table 3. Oviposition profiles of intact, dsbla-injected and dsC/kinjected crickets.

\begin{tabular}{lcccccccc}
\hline \multirow{2}{*}{ Photoperiod } & Treatment & $n$ & \multicolumn{7}{c}{\begin{tabular}{c} 
Oviposition profile types \\
\cline { 3 - 7 }
\end{tabular}} & & 1 & 2 & 3 & 4 & 5 & Statistical \\
test
\end{tabular}

$\S$ Type 1: incidence of diapause is always higher than $50 \%$. Type 2: incidence of diapause is lower than $50 \%$ at the beginning of the experimental period, is higher than $50 \%$ during the middle period and is subsequently lower than $50 \%$ at the end of the period. Type 3 : incidence of diapause is lower than $50 \%$ at the beginning of the experimental period but higher than $50 \%$ during the middle or end of the period. Type 4: incidence of diapause is initially higher than $50 \%$ but later becomes lower than 50\%. Type 5: incidence of diapause is lower than $50 \%$ throughout the experimental period. See, Fig. 1. \#Different letters indicate a significant difference (Mann-Whitney $U$ and Steel-Dwass tests for short and long days, respectively; $P<$ 0.05). 
Short days, intact
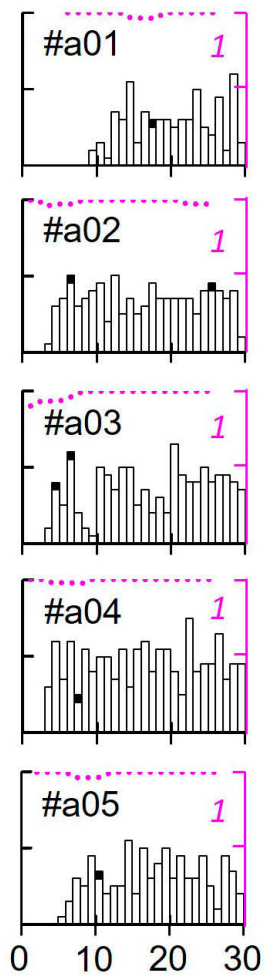

Short days, dsbla
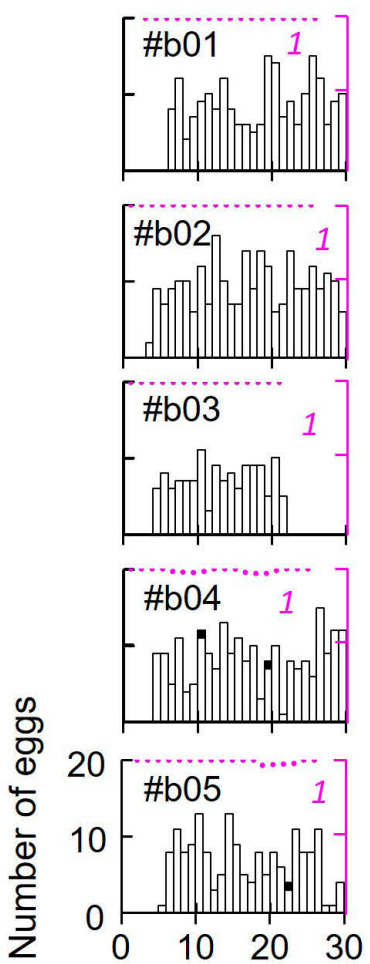

Days after injection
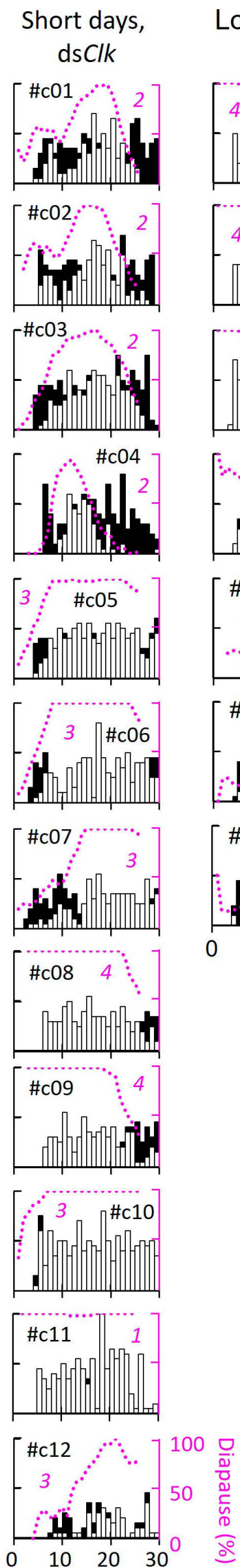

18

$\begin{array}{ccc}\text { Long days, } & \begin{array}{c}\text { Long days, } \\ \text { intact }\end{array} & \begin{array}{c}\text { Long days, } \\ \text { ds } C l k\end{array}\end{array}$
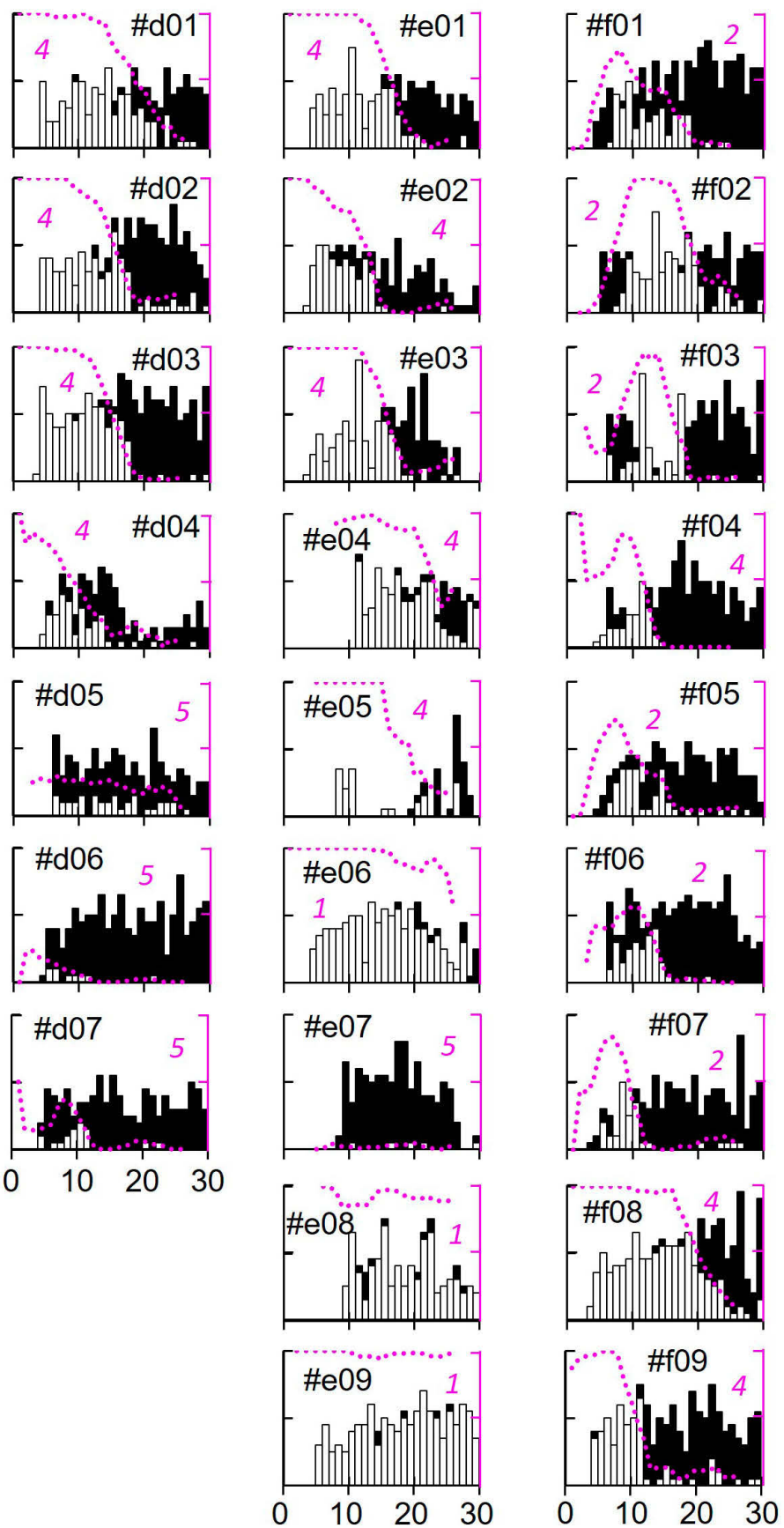

Diapause

Non-diapause

Fig. 5. Temporal changes in the number of diapause- (open columns) and non-diapause-(closed columns) type eggs laid by intact, dsbla-injected and dsClk-injected females of Dianemobius nigrofasciatus under short-day and long-day conditions. The 5-day moving average of the incidence of diapause is shown in violet. The types of the temporal profiles in the incidence of diapause are also shown in violet (see Fig. 1 for details). Individual identification numbers are shown in black. 


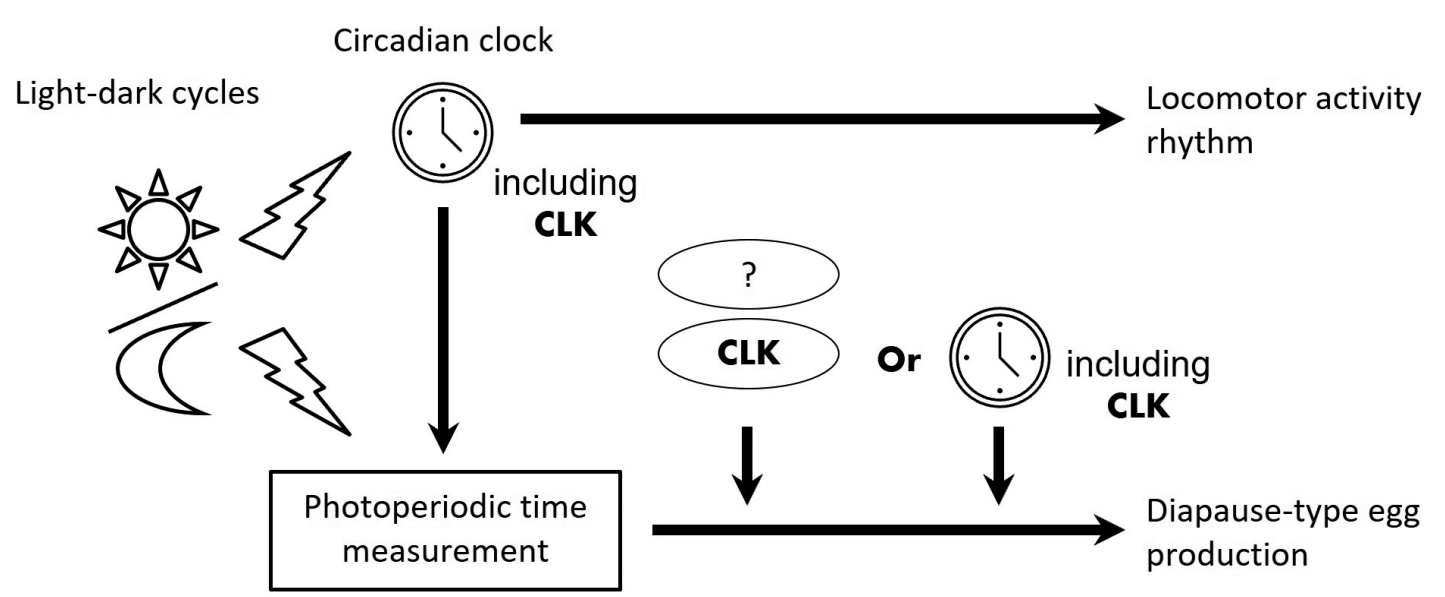

Fig. 6. Roles of CLOCK (CLK) in the maternal photoperiodic regulation of embryonic diapause in Dianemobius nigrofasciatus. CLK is involved not only in photoperiodic time measurement but also in its downstream process controlling the production of diapause-type eggs. It is still uncertain whether the circadian clock or CLK independently of the circadian clock are involved in the downstream processes.

als, with some crickets responding immediately to long days, whereas for others, a period of 10 days or more was necessary for females to alter the developmental trajectory of their offspring. We observed that dsbla-injected crickets showed a similar response, although three crickets failed to respond to long days and continued to lay diapause-type eggs (Type 1).

The oviposition profiles of dsClk-injected crickets that were continuously reared under short-day conditions differed from those of intact and dsbla-injected crickets (Fig. 5 and Table 3), with nine of 12 crickets immediately depositing non-diapause-type eggs. Among these, five crickets subsequently changed the developmental trajectory of their offspring from non-diapause to diapause (i.e. Type 3). The remaining four similarly altered the developmental trajectory from non-diapause to diapause, although subsequently reversed the trajectory from diapause to non-diapause (i.e. Type 2). Furthermore, two crickets showed a Type 4 oviposition profile. Only one female was characterised by the same oviposition type as intact and dsbla-injected crickets under the same photoperiodic conditions (i.e. Type 1). In Clk RNAi crickets transferred from short-day to long-day conditions, the oviposition profiles of six crickets were categorised as Type 2, a type not recorded in intact and dsbla-injected crickets under long days. The remaining three females showed Type 4 behaviour. Statistically, the oviposition profiles of $C l k$ RNAi crickets were found to differ significantly from those of control individuals (Table 3; Mann-Whitney $U$-test for short days, $P<0.05$; SteelDwass test for long days, $P<0.05$ ).

\section{DISCUSSION}

The putative amino acid sequence of the CLK of $D$. nigrofasciatus is very similar to that of the CLK of other insects and contains PAS domains, which play an important role in dimerization of the CLK with its partner protein CYC (Allada et al., 1998; Darlington et al., 1998). We detected no or little variation in the level of $C l k$ mRNA in $D$. nigrofasciatus kept under light-dark conditions, which is consistent with the observations reported for various other insects, including crickets (Rubin et al., 2006; Kamae et al., 2010; Moriyama et al., 2012; Ikeno et al., 2013; Takekata et al., 2014; Meuti et al., 2015; Barberà et al., 2017). Moreover, as reported for other species of crickets (Moriyama et al., 2012; Takekata et al., 2014) the injection of Clk dsRNA suppressed the expression of Clk in D. nigrofasciatus.

We confirmed that the locomotor activity of $D$. nigrofasciatus is clearly rhythmical, both in constant darkness and under light-dark conditions, as reported previously (Shiga et al., 1999). Moreover, Clk RNAi disrupted the circadian rhythm of locomotor activity under constant darkness and LD conditions, but the rhythmicity of $C l k$ RNAi crickets with a malfunctioning circadian clock was incomplete or exhibited the masking effect in their locomotor activity, which is consistent with the reports for other species of crickets (Sakamoto et al., 2009; Moriyama et al., 2012).

We confirmed that maternal regulation of diapause occurs in D. nigrofasciatus, as reported previously (Shiga \& Numata, 1996). Control females reared continuously under short days laid eggs destined to diapause throughout the experimental period, whereas in contrast, control females that were maintained under short-day conditions and subsequently transferred to long days, laid non-diapause-type eggs. However, Clk RNAi crickets showed distinct oviposition profiles, with more than half of those reared under short days immediately laid non-diapause-type eggs (Types 2 and 3) and the remainder laid non-diapause-type eggs on day 15 or later (Types 2 and 4). These profiles were not recorded for short-day control insects. Under longday conditions, more than half of the Clk RNAi crickets immediately laid non-diapause-type eggs, as recorded for short-day Clk RNAi crickets. Similar to long-day control insects, all $C l k$ RNAi crickets showed a reduction in the production of eggs destined to diapause during the course of the experiment.

Involvement of a circadian clock in photoperiodic time measurement was previously proposed by several authors (Pegoraro et al., 2014; Mukai \& Goto, 2016; Urbanová et al., 2016; Ikeda et al., 2021), among whom, Pegoraro et al. (2014) focused on the photoperiodic modulation of chill- 
coma recovery time (CCRt) in Drosophila melanogaster. Wild-type flies raised under short days exhibit significantly shorter CCRts than flies maintained under long days, whereas mutants with a long free-running period (e.g. slow circadian clock) consistently showed shorter CCRts under both long- and short-day photoperiods than mutants with a short free-running period (e.g. fast circadian clock). The circadian period of the flies was accordingly found to determine the photoperiodic CCRt, thereby indicating the involvement of the circadian clock in photoperiodic time measurement. Mukai \& Goto (2016) reveal that per RNAi disrupts photoperiodic induction of diapause in the parasitic wasp Nasonia vitripennis, although per RNAi wasps are still able to enter diapause in response to chilling. Furthermore, in the linden bug P. apterus, Clk RNAi has no significant effects on the reproduction of males under long days, although it disrupted the ability of diapausing males to switch to a reproductive mode following transfer to long days (Urbanová et al., 2016). These findings thus provide convincing evidence of the role of the circadian clock in photoperiodic time measurement.

Prior to oviposition, the females of D. nigrofasciatus are assumed to monitor and assess photoperiodic patterns for several days and use this information to determine the developmental trajectory of their offspring. Consequently, there is a lag of several days or even weeks between photoperiodic perception and actual changes in the incidence of diapause (Kidokoro \& Masaki, 1978). The laying of nondiapause-type eggs by late-stage female $C l k$ RNAi crickets under short days appears to be similar to that of control crickets under long days. Collectively, these results tend to indicate that CLK is involved in photoperiodic time measurement in D. nigrofasciatus (Fig. 6).

Our observation of the immediate laying of non-diapause-type eggs by $C l k$ RNAi crickets under short-day conditions is believed to be unique. Such immediate effects would be difficult to explain in terms of the dysfunction of a circadian clock involved in photoperiodic time measurement. This immediate response might, nevertheless, indicate the involvement of $C l k$ in the production of diapausetype eggs. It is, however, uncertain as to why the immediate effect of producing non-diapause-type eggs did not persist in Clk RNAi crickets throughout the experimental period in the present study, although a plausible explanation is that with respect to the laying of diapause-type eggs, the RNAi effect persists only for a few days. Moreover, it has yet to be ascertained whether the circadian clock including CLK or CLK independently of the circadian clock (gene pleiotropy; Emerson et al., 2009) regulates the production of diapause-type eggs (Fig. 6). The non-clock function of the circadian clock gene in inducing diapause is proposed for several species. In the gut of $P$. apterus, which enters reproductive diapause, $c r y-m$ and $P d p 1_{i s o l}$ (an isoform of $P d p 1)$ are inversely regulated under diapause and reproductive conditions; the former remains high in diapausing but low in reproductive females, while the latter remains high in reproductive but low in females in diapause, with no daily oscillation. Their expression is indispensable for diapause-/non-diapause-specific gene expression in the gut. It is noteworthy that the expression of $c r y-m$ and $P d p 1_{\text {iso }}$ is controlled by $C l k$ and $c y c$ and also by juvenile hormone, which directly determines the diapause/non-diapause phenotype, suggesting a noncircadian function downstream of photoperiodic time measurement (Bajgar et al., 2013b). In Drosophila triauraria, geographic variation exists in the occurrence of diapause. A strain from high latitude enters reproductive diapause in response to short days, while strains from low latitudes failed to enter diapause and reproduce irrespective of photoperiod. Single nucleotide polymorphism and deletion analyses of five genes (per, tim, $C l k, c y c$ and $c r y-d$ ) in backcross progeny reveal that allelic differences in tim and cry- $d$ are 'additively' associated with differences in the incidence of diapause. Their independent effects suggest that the incidence of diapause is not based on a circadian clock (Yamada \& Yamamoto, 2011).

In conclusion, the findings of the present study provide clear evidence that indicate the clock gene $C l k$ is involved not only in photoperiodic time measurement, but also in the production of diapause-type eggs. In this regard, one area for future research should be a physiological dissection of the photoperiodic time measurement system. Although there are many theoretical models of photoperiodic time measurement (Vaz Nunes \& Saunders, 1999), the underlying molecular machinery has yet to be determined. The system is assumed to be associated with or inherent to the circadian clock, and the anatomical locations of the circadian clock in D. nigrofasciatus have been verified (Shao et al., 2006, 2008). Characterizing the circadian clock neurons and output molecules contributing to the control of photoperiodic responses should be the main focus of the molecular dissection of the photoperiodic time measurement system. A further goal of future research is the unravelling of the process controlling the production of diapause-type eggs, in which $C l k$ is believed to be involved. The role of other clock genes should also be investigated on order to clarify whether the circadian clock as a whole or individual genes are involved in the production of diapause-type eggs. In Bombyx mori, diapause hormone, an FXPRL amide is the endocrine effector that programmes embryonic diapause (Ikeda et al., 1993) and a recent study has revealed that silencing the per gene in $B$. mori results in diapause via a GABAeric-diapause hormone pathway (Cui et al., 2021). Furthermore, in the locust Locusta migratoria maternal regulation of embryonic diapause is governed by the hormone and genes related to the forkhead box $\mathrm{O}$ (FOXO) cascade (Hao et al., 2019ba, b). Thus it would be of considerable interest to establish whether similar cascades are involved in diapause in D. nigrofasciatus and elucidate the role of $C l k$ in this process.

ACKNOWLEDGEMENTS. We dedicate this paper to the memory of I. Hodek, whose friendship and insights into insect life cycles and their regulatory mechanisms we have highly valued. We thank T. Shizuka for preparing the figures. We also acknowledge Editage (www.editage.co.jp) for English corrections. This study was supported in part by JSPS KAKENHI Grant No. 25450488. 


\section{REFERENCES}

Allada R., White N.E., So W.V., Hall J.C. \& Rosbash M. 1998 A mutant Drosophila homolog of mammalian Clock disrupts circadian rhythms and transcription of period and timeless. Cell 93: 791-804.

Bajgar A., Dolezel D. \& Hodkova M. 2013a: Endocrine regulation of non-circadian behavior of circadian genes in insect gut. — J. Insect Physiol. 59: 881-886.

Bajgar A., JindRa M. \& Dolezel D. 2013b: Autonomous regulation of the insect gut by circadian genes acting downstream of juvenile hormone signaling. - Proc. Natl. Acad. Sci. U.S.A. 110: 4416-4421.

Barberà M., Collantes-Alegre J.M. \& Martínez-Torres D. 2017: Characterisation, analysis of expression and localisation of circadian clock genes from the perspective of photoperiodism in the aphid Acyrthosiphon pisum. - Insect Biochem. Mol. Biol. 83: 54-67.

Bradshaw W.E. \& Holzapfel C.M. 2010: Circadian clock genes, ovarian development and diapause. - BMC Biol. 8: 115, 4 pp.

BüNNING E. 1936: Die endogene Tagesrhythmik als Grundlage der photoperiodischen Reaktion. - Ber. Dtsch. Bot. Ges. $\mathbf{5 4}$ 590-607.

Cui W.-Z., Qiu J.-F., Dai T.-M., Chen Z., Li J.-L., Liu K., Wang Y.-J., SimA Y.-H. \& XU S.-Q. 2021: Circadian clock gene period contributes to diapause via GABAeric-diapause hormone pathway in Bombyx mori. - Biology (Basel) 10: 842, 20 pp.

Danks H.V. 1987: Insect Dormancy: An Ecological Perspective. Biological Survey of Canada, Ottawa, $439 \mathrm{pp}$.

Darlington T.K., Wager-Smith K., Ceriani M.F., Staknis D., GeKakis N., Steeves T.D.L., Weitz C.J., Takahashi J.S. \& KAY S.A. 1998: Closing the circadian loop: CLOCK-induced transcription of its own inhibitors per and tim. - Science $\mathbf{2 8 0}$ : 1599-1603.

Denlinger D.L., Yocum G.D. \& Rinehart J.P. 2012: Hormonal control of diapause. In Gilbert L.I. (ed.): Insect Endocrinology. Elsevier, Amsterdam, pp. 430-463.

Emerson K.J., Bradshaw W.E. \& Holzapfel C.M. 2009: Complications of complexity: integrating environmental, genetic and hormonal control of insect diapause. - Trends Genet. 25: $217-225$.

Goto S.G., Doi K., NaKayama S. \& Numata H. 2008: Maternal control of cold and desiccation tolerance in eggs of the bandlegged ground cricket Dianemobius nigrofasciatus in relation to embryonic diapause. - Entomol. Res. 38: 17-23.

Hao K., Jarwar A.R., Ullah H., Tu X., Nong X. \& Zhang Z. 2019a: Transcriptome sequencing reveals potential mechanisms of the maternal effect on egg diapause induction of Locusta migratoria. - Int. J. Mol. Sci. 20: 1974, 19 pp.

Hao K., Tu X., Ullah H., McNeill M.R. \& Zhang Z. 2019b: Novel Lom- $d h$ genes play potential role in promoting egg diapause of Locusta migratoria L. - Front. Physiol. 10: 767, 14 pp.

Iiams S.E., Lugena A.B., Zhang Y., Hayden A.N. \& Merlin C. 2019: Photoperiodic and clock regulation of the vitamin A pathway in the brain mediates seasonal responsiveness in the monarch butterfly. - Proc. Natl. Acad. Sci. U.S.A. 116: 25214-25221.

Ikeda M., Su Z., Saito H., Imai K., Sato Y., Isobe M. \& YamashiTA O. 1993: Induction of embryonic diapause and stimulation of ovary trehalase activity in the silkworm, Bombyx mori, by synthetic diapause hormone. - J. Insect Physiol. 39: 889-895.

Ikeda K., Daimon T., Shiomi K., Udaka H. \& Numata H. 2021: Involvement of the clock gene period in the photoperiodism of the silkmoth Bombyx mori. — Zool. Sci. 38: 523-530.
Ikeno T., Tanaka S.I., Numata H. \& Goto S.G. 2010: Photoperiodic diapause under the control of circadian clock genes in an insect. - BMC Biol. 8: 116, 9 pp.

IKeno T., Numata H. \& Goto S.G. 2011a: Circadian clock genes period and cycle regulate photoperiodic diapause in the bean bug Riptortus pedestris males. - J. Insect Physiol. 57: 935938.

Ikeno T., Numata H. \& Goto S.G. 2011b: Photoperiodic response requires mammalian-type cryptochrome in the bean bug Riptortus pedestris. - Biochem. Biophys. Res. Commun. 410: 394-397.

Ikeno T., Ishikawa K., Numata H. \& Goto S.G. 2013: Circadian clock gene Clock is involved in the photoperiodic response of the bean bug Riptortus pedestris. - Physiol. Entomol. 38: $157-162$.

Kamae Y., Tanaka F. \& Tomioka K. 2010: Molecular cloning and functional analysis of the clock genes, Clock and cycle, in the firebrat Thermobia domestica. - J. Insect Physiol. 56: 1291-1299.

Kidokoro T. \& MASAKI S. 1978: Photoperiodic response in relation to variable voltinism in the ground cricket, Pteronemobius fascipes Walker (Orthoptera: Gryllidae). — Japan. J. Ecol. 28: 291-298.

Kilman V.L. \& Allada R. 2009: Genetic analysis of ectopic circadian clock induction in Drosophila. - J. Biol. Rhythms 24: 368-378.

KošŤÁL V. 2006: Eco-physiological phases of insect diapause. J. Insect Physiol. 52: 113-127.

Kotwica-Rolinska J., Pivarciova L., Vaneckova H. \& Dolezel D. 2017: The role of circadian clock genes in the photoperiodic timer of the linden bug Pyrrhocoris apterus during the nymphal stage. - Physiol. Entomol. 42: 266-273.

Letunic I., KHedKar S. \& Bork P. 2021: SMART: recent updates, new developments and status in 2020. - Nucl. Acids Res. 49: D458-D460.

MASAKI S. 1960: Thermal relations of diapause in the eggs of certain crickets (Orthoptera: Gryllidae). - Bull. Fac. Agric. Hirosaki Univ. 6: 5-20.

Meuti M.E., Stone M., Ikeno T. \& Denlinger D.L. 2015: Functional circadian clock genes are essential for the overwintering diapause of the Northern house mosquito, Culex pipiens. - J. Exp. Biol. 218: 412-422.

Mohamed A.A.M., Wang Q., Bembenek J., Ichihara N., Hiragaki S., SuzUKI T. \& TAKedA M. 2014: N-acetyltransferase (nat) is a critical conjunct of photoperiodism between the circadian system and endocrine axis in Antheraea pernyi. — PLOS ONE 9: e92680, 14 pp.

Moriyama Y., Kamae Y., Uryu O. \& Tomioka K. 2012: Gb’ Clock is expressed in the optic lobe and is required for the circadian clock in the cricket Gryllus bimaculatus. - J. Biol. Rhythms 27: 467-477.

Mukai A. \& Goto S.G. 2016: The clock gene period is essential for the photoperiodic response in the jewel wasp Nasonia vitripennis (Hymenoptera: Pteromalidae). - Appl. Entomol. Zool. 51: 185-194.

Patke A., Young M.W. \& Axelrod S. 2020: Molecular mechanisms and physiological importance of circadian rhythms. Nat. Rev. Mol. Cell Biol. 21: 67-84.

Pegoraro M., Gesto J.S., Kyriacou C.P. \& Tauber E. 2014: Role for circadian clock genes in seasonal timing: Testing the Bünning hypothesis. - PLoS Genet. 10: e1004603, 9 pp.

Rubin E.B., Shemesh Y., Cohen M., Elgavish S., Robertson H.M. \& BLOCH G. 2006: Molecular and phylogenetic analyses reveal mammalian-like clockwork in the honey bee (Apis mellifera) 
and shed new light on the molecular evolution of the circadian clock. - Genome Res. 16: 1352-1365.

Sakamoto T., URyu O. \& Tomioka K. 2009: The clock gene period plays an essential role in photoperiodic control of nymphal development in the cricket Modicogryllus siamensis. - J. Biol. Rhythms 24: 379-390.

SAUNDERS D.S. 2020: Dormancy, diapause, and the role of the circadian system in insect photoperiodism. - Annu. Rev. Entomol. 65: 373-389.

Saunders D.S., Henrich V.C. \& Gilbert L.I. 1989: Induction of diapause in Drosophila melanogaster: photoperiodic regulation and the impact of arrhythmic clock mutations on time measurement. - Proc. Natl. Acad. Sci. U.S.A. 86: 3748-3752.

Shao Q.M., Bembenek J., Trang L.T.D., Hiragaki S. \& Takeda M. 2008: Molecular structure, expression patterns, and localization of the circadian transcription modulator CYCLE in the cricket, Dianemobius nigrofasciatus. - J. Insect Physiol. 54: 403-413.

Shao Q.M., Sehadová H., Ichihara N., Sehnal F. \& Takeda M. 2006: Immunoreactivities to three circadian clock proteins in two ground crickets suggest interspecific diversity of the circadian clock structure. - J. Biol. Rhythms 21: 118-131.

Shiga S. \& Numata H. 1996: Effects of compound eye-removal on the photoperiodic response of the band-legged ground cricket, Pteronemobius nigrofasciatus. - J. Comp. Physiol. (A) 179: 625-633.

Shiga S. \& Numata H. 1997: Seasonal changes in the incidence of embryonic diapause in the band-legged ground cricket, $\mathrm{Di}$ anemobius nigrofasciatus. - Zool. Sci. 14: 1015-1018.

Shiga S., Numata H. \& Yoshioka E. 1999: Localization of the photoreceptor and pacemaker for the circadian activity rhythm in the band-legged ground cricket, Dianemobius nigrofasciatus. - Zool. Sci. 16: 193-201.

Sokolove P.G. \& Bushell W.N. 1978: The chi square periodogram: Its utility for analysis of circadian rhythms. $-J$. Theor. Biol. 72: 131-160.

TakeKata H., Numata H., Shiga S. \& Goto S.G. 2014: Silencing the circadian clock gene Clock using RNAi reveals dissociation of the circatidal clock from the circadian clock in the mangrove cricket. - J. Insect Physiol. 68: 16-22.
Tamai T., Shiga S. \& Goto S.G. 2019: Roles of the circadian clock and endocrine regulator in the photoperiodic response of the brown-winged green bug Plautia stali. - Physiol. Entomol. 44: 43-52.

Tanigawa N., Matsumoto K., Yasuyama K., Numata H. \& Shiga S. 2009: Early embryonic development and diapause stage in the band-legged ground cricket Dianemobius nigrofasciatus. - Dev. Genes Evol. 219: 589-596.

TAuber M.J., TAuber C.A. \& MASAKI S. 1986: Seasonal Adaptations of Insects. Oxford University Press, Oxford, 416 pp.

Tauber E., Zordan M., Sandrelli F., Pegoraro M., Osterwalder N., Breda C., Daga A., Selmin A., Monger K., Benna C., et AL. 2007: Natural selection favors a newly derived timeless allele in Drosophila melanogaster. — Science 316: 1895-1898.

Томіока K. 2014: Chronobiology of crickets: a review. - Zool. Sci. 31: 624-632.

Tоміока K. \& Mатsumoto A. 2015: Circadian molecular clockworks in non-model insects. - Curr. Opin. Insect Sci. 7: 5864.

Urbanová V., Bazalová O., VaněčKová H. \& Doležel D. 2016 : Photoperiod regulates growth of male accessory glands through juvenile hormone signaling in the linden bug, Pyrrhocoris apterus. - Insect Biochem. Mol. Biol. 70: 184-190.

Vaz Nunes M. \& Saunders D. 1999: Photoperiodic time measurement in insects: A review of clock models. - J. Biol. Rhythms 14: 84-104.

Yamada H. \& Yамамото M.T. 2011: Association between circadian clock genes and diapause incidence in Drosophila triauraria. - PLoS ONE 6: e27493, 9 pp.

Zhao J., Kilman V.L., Keegan K.P., Peng Y., Emery P., Rosbash M. \& Allada R. 2003: Drosophila clock can generate ectopic circadian clocks. - Cell 113: 755-766.

Zhu L., Tian Z., Guo S., Liu W., Zhu F. \& Wang X.P. 2019: Circadian clock genes link photoperiodic signals to lipid accumulation during diapause preparation in the diapause-destined female cabbage beetles Colaphellus bowringi. - Insect Biochem. Mol. Biol. 104: 1-10.

Received November 4, 2021; revised and accepted December 15, 2021 Published online January 7, 2022 